\title{
Two-Dimensional Communication of Networked Devices Through a Single Conductive Surface
}

\author{
Josep Rius
}

\begin{abstract}
This paper shows the possibility of two-dimensional communication between electronic devices by using only one conductive surface. An analysis of the expected magnitude of the signal at the reception point as a function of distance and electrical parameters of the conductive surface is presented. Measurements on surfaces from 0.4 to $2.56 \mathrm{~m}^{2}$ and with sheet resistances from 77 to $1462 \mathrm{ohm} /$ square prove the correctness of this analysis. In addition, practical rules to locate and orient the transceivers are given. Prototype transceivers connected to a plane with one conductive surface able to communicate at $100 \mathrm{Kbit} / \mathrm{s}$ at a distance of $2 \mathrm{~m}$ are described.
\end{abstract}

Index Terms - computer networks, conducting films, data communication, distributed parameter circuits.

\section{INTRODUCTION}

$\mathrm{M}$ OST data communication applications are generally carried out by one-dimensional (wiring cables, interconnects, or fibers) or three-dimensional (wireless transmission through free space, gases or liquids) media, whereas communication by two-dimensional media is less considered. Surfaces, however, are ubiquitous in everyday life. Walls, tables, garments and floors are a few examples of the pervasive presence of surfaces. This ubiquity offers unique opportunities for electronic communication in applications where ordinary wire or wireless technologies may appear less attractive.

Communication through conductive surfaces has some of the benefits of wire and wireless technologies like easy networking, two-dimensional mobility of transceivers, power and signal paths and it is potentially not electrically disturbing. Moreover, this type of communication provides a robust connection between devices because there are multiple signal paths.

In the early years of this decade, several papers presented the concept of data transmission through two-dimensional media [1][2][4][6] and in the following years applications of this concept were published in the field of networked sensors

This work was supported in part by the Comisión Interministerial para la Ciencia y la Tecnología under Project TEC2007-66672, and the Spanish Secretaría de Estado de Educación, Universidades y Desarrollo.

J. Rius is with the Dept. d'Enginyeria Electrònica of Universitat Politècnica de Catalunya, 08028 Barcelona, Spain, (phone: 34934016650 , fax: 34 934017785; email: rius@eel.upc.edu).
[3-5], [7-9] and other fields such as clothing [10-12] and medicine $[13,14]$.

These proposals have in common that data transmission is always performed through a pair of conductive surfaces. Data propagate as an electromagnetic wave in the dielectric layer insulating the two conductive layers (or as a diffusive perturbation [6]) and are finally detected at the receiver point. Two conductive planes must be available, implying that: (a) a relatively complex and expensive fabrication process to generate and join the conductive layers at both sides of the insulation is involved (b) careful control during fabrication is required to avoid short-circuits between the conductive layers, (c) an unintentional short-circuit between the two conductive layers during operation may degrade or interrupt the communication, and (d) the interface between the electronic devices and both surfaces is relatively complex.

In contrast, data communication by a single conductive surface has the following benefits: (a) simpler and less expensive fabrication process because only one conductive layer is needed, (b) short-circuits are not an issue, and (c) the interface between the electronic devices and a single conductive layer is much less complex.

This approach (which, as far as the author knows, has not been previously proposed) is sketched in Figure 1. The signal from the transmitter $\mathbf{T}$ (we call it transmitter dipole) is a timevarying potential difference between two points of the single conductive sheet, namely $x_{T 1}, y_{T 1}$ and $x_{T 2}, y_{T 2}$., which generates a time-varying, two-dimensional potential field on the conductive sheet that propagates to the receiver $\mathbf{R}$ (we call it receiver dipole), which is connected to the conductive sheet at points $x_{R 1}, y_{R 1}$ and $x_{R 2}, y_{R 2}$. The potential difference between these two points, $V_{R}$, is the received signal.

The parameters of interest are the sheet resistance $R_{S}$ and the parasitic capacitances (not shown in the Figure) between the conductive sheet and the surrounding bodies (Earth, metallic bodies, etc).

The goal of this paper is to show the feasibility of data transmission between networked devices using only one conductive surface as the transmission medium. Furthermore, the expected amplitude of the received signal as a function of plane dimensions, electrical parameters and transmitter and receiver sizes and distances, as well as the implications of this approach for data transmission in terms of speed and power consumption of the connected devices are analyzed. 


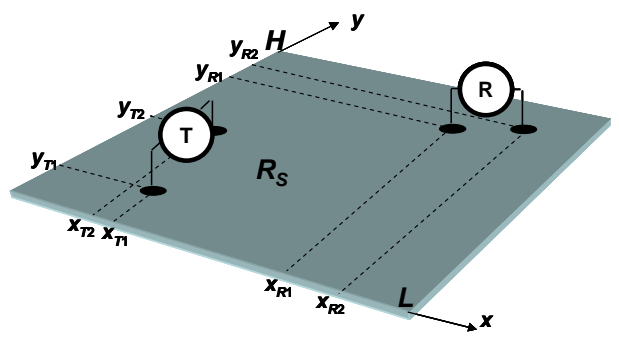

Fig. 1. Plane with only one conductive surface.

The paper is organized as follows: Section 2 derives formulas to calculate the voltage at the receiver dipole when the transmitter dipole injects/withdraws a constant current elsewhere. Section 3 analyzes the same problem when the excitation is a time-varying current. In this case, the analysis is extended to planes with one and two conductive surfaces. Section 4 provides experimental results obtained from several samples of planes with one and two conductive surfaces and compares them with the analytical results of Sections 2 and 3. It also presents a prototype implementation of a communication system built on one of the samples. In Section 5 , the main relationships between sheet resistance, power consumption, speed and dimensions of the communication system are investigated. Finally, Section 6 summarizes the work and draws conclusions.

\section{STATIC ANALYSIS}

In static conditions, the potential difference $V_{R}$ at the receiver electrodes is independent of time. This potential difference as a function of the surface size and shape and the physical parameters can often be calculated by numerical methods only. However, for simple geometries, like rectangular ones, it is possible to derive closed form expressions for $V_{R}$. This is the goal of this Section.

The problem can be divided into three parts, namely obtaining the potential at any single point on the surface of an infinite isolated conductive strip caused by two filament lines which inject and withdraw a constant current into and from the strip at two given points; generalizing this result to a rectangular surface, and finally deriving a closed form expression for the potential difference (voltage) between two arbitrary points on the rectangular surface.

\section{A. Potential at any point on an infinite isolated strip caused by the injection/withdrawal of current at two arbitrary points}

Figure 2 sketches the geometry of the problem. We first derive a formula for the potential at a point $P$ produced by two current filaments of infinite length and constant magnitude $I$ and $-I$ at points $(a, 0)$ and $(b, 0)$ in a conductive medium limited by two surfaces $\mathrm{A}$ and $\mathrm{B}$ of infinite extent in $y$ and $z$ directions. With these conditions, the problem becomes two-dimensional and the region of interest is the strip of width $L$ and infinite length limited by lines A and B. The current enters the strip at point $(a, 0)$ and leaves it at point $(b, 0)$. No current crosses A and $B$. The electrical parameter defining the conductive medium in the strip is its sheet resistance $R_{S}$.

Let the origin of the complex plane be 0 , and $P$ the point $z=$ $x+j y$. The images of the current lines are shown at points $z_{1+}$, $z_{-1+}, z_{2+}, z_{-2+}, \ldots$ and $z_{1-}, z_{-1-}, z_{2-}, z_{-2-}$, etc. whose distances from $P$ are $r_{1+}, r_{-1+}, r_{2+}, r_{-2+}, \ldots$ and $r_{1-}, r_{-1-}, r_{2-}, r_{-2-}$, etc. The potential at $P$ caused by the currents at $a$ and $b$ and the induced images will be calculated from the current itself and its infinite set of images. The total potential at $P$ is a sum of expressions, one for each current, as shown by equation (1):

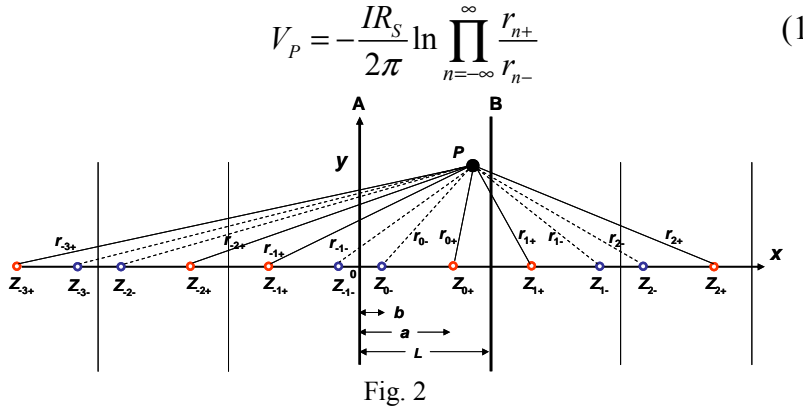

According to [15] and [16], we can develop the infinite product of (1) to obtain the following closed form expression:

$$
V_{P}=\frac{-I R_{S}}{2 \pi} \operatorname{Re}\left\{\ln \frac{\sin \left(\pi \frac{z+a}{2 L}\right) \sin \left(\pi \frac{z-a}{2 L}\right)}{\sin \left(\pi \frac{z+b}{2 L}\right) \sin \left(\pi \frac{z-b}{2 L}\right)}\right\}
$$

where $\operatorname{Re}\{\cdot\}$ denotes the real part. Now, in order to find the potential in a rectangular plane, we need to generalize this result.

\section{B. Potential in a rectangular plane}

Imagine a pair of filament wires of infinite length intersecting a perpendicular rectangular plane of dimensions $L$, $H$ at points $z_{+}=x_{+}+j y_{+}$and $z_{-}=x_{-}+j y_{-}$. The wires inject and withdraw a constant current $I$ and $-I$ into and from the plane, respectively. Let the origin of the complex plane be identified as $0, P$ the point $z=x+j y$ and the four sides of the plane be isolated. For the case of the rectangular plane the potential at $P$ can be obtained by generalizing formula (2) in the previous sub-section.

In this case there is an infinite number of horizontal rows of images for each current which must be considered to obtain the desired boundary conditions of zero current at the sides of the rectangle of vertices $(0,0),(L, 0),(L, H)$ and $(0, H)$.

Therefore, the total potential at point $P$ results from the addition of eight contributions: four due to an infinite number of horizontal rows of images of the positive current, and four due to an infinite number of horizontal rows of images of the negative current. The double periodicity of the images suggests the use of elliptic functions [16] to obtain $V_{P}$. The result is the following closed form equation (3):

$$
V_{P}(z)=\frac{-I R_{S}}{2 \pi} \operatorname{Re}\left\{\ln \frac{\vartheta_{1}\left(\pi \frac{z-z_{+}}{2 L}\right) \cdot \vartheta_{1}\left(\pi \frac{z+z_{+}}{2 L}\right) \cdot \vartheta_{1}\left(\pi \frac{z-\bar{z}_{+}}{2 L}\right) \cdot \vartheta_{1}\left(\pi \frac{z+\bar{z}_{+}}{2 L}\right)}{\vartheta_{1}\left(\pi \frac{z-z_{-}}{2 L}\right) \cdot \vartheta_{1}\left(\pi \frac{z+z_{-}}{2 L}\right) \cdot \vartheta_{1}\left(\pi \frac{z-\bar{z}_{-}}{2 L}\right) \cdot \vartheta_{1}\left(\pi \frac{z+\bar{z}_{-}}{2 L}\right)}\right\}
$$

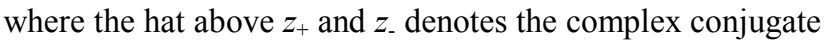
and the elliptic theta function $\vartheta_{1}(\cdot)$ with parameter $q=\exp (-$ 
$\pi H / L$ ) is a standard built-in function (like trigonometric ones) in any computer algebra system.

\section{Potential difference at the receiver dipole}

Now the potential difference $V_{R}$ between points R1 and R2 of Figure 1 can be calculated as the real part of the difference between two logarithms. That is,

$$
V_{R}(z)=\frac{-I R_{S}}{2 \pi} \operatorname{Re}\left\{\begin{array}{c}
\left(\begin{array}{c}
\left.\ln \frac{\vartheta_{1}\left(\frac{\pi}{2 L}\left(z-z_{+}\right)\right) \cdot \vartheta_{1}\left(\frac{\pi}{2 L}\left(z+z_{+}\right)\right) \cdot \vartheta_{1}\left(\frac{\pi}{2 L}\left(z-\bar{z}_{+}\right)\right) \cdot \vartheta_{1}\left(\frac{\pi}{2 L}\left(z+\bar{z}_{+}\right)\right)}{\vartheta_{1}\left(\frac{\pi}{2 L}\left(z-z_{-}\right)\right) \cdot \vartheta_{1}\left(\frac{\pi}{2 L}\left(z+z_{-}\right)\right) \cdot \vartheta_{1}\left(\frac{\pi}{2 L}\left(z-\bar{z}_{-}\right)\right) \cdot \vartheta_{1}\left(\frac{\pi}{2 L}\left(z+\bar{z}_{-}\right)\right)}\right)_{z=z_{R_{1}}} \\
\quad-\left(\begin{array}{l}
\vartheta_{1}\left(\frac{\pi}{2 L}\left(z-z_{+}\right)\right) \cdot \vartheta_{1}\left(\frac{\pi}{2 L}\left(z+z_{+}\right)\right) \cdot \vartheta_{1}\left(\frac{\pi}{2 L}\left(z-\bar{z}_{+}\right)\right) \cdot \vartheta_{1}\left(\frac{\pi}{2 L}\left(z+\bar{z}_{+}\right)\right) \\
\vartheta_{1}\left(\frac{\pi}{2 L}\left(z-z_{-}\right)\right) \cdot \vartheta_{1}\left(\frac{\pi}{2 L}\left(z+z_{-}\right)\right) \cdot \vartheta_{1}\left(\frac{\pi}{2 L}\left(z-\bar{z}_{-}\right)\right) \cdot \vartheta_{1}\left(\frac{\pi}{2 L}\left(z+\bar{z}_{-}\right)\right)
\end{array}\right)
\end{array}\right)_{z=z_{R_{2}}}
\end{array}\right.
$$

Expression (4) is the final closed form solution of the potential difference at the receiver electrodes when the transmitter dipole injects/withdraws a constant current.

\section{DYNAMIC ANALYSIS}

When a time-varying current is applied at the transmitter electrodes, the signal captured by the receiver has a smaller amplitude than in the static case. The purpose of this section is to obtain expressions for this amplitude as a function of the electrical parameters and dimensions of the rectangle, and as a function of the signal frequency.

\section{A. Solution for an infinite plane}

Let us first find the potential at a point $P$ placed at distance $r$ from the origin of an infinite plane when a steady-state sinusoidal current $I_{0} \exp (j \omega t)$ is injected at the origin. The plane has the following parameters: sheet resistance, $R_{S}[\Omega /$ square $]$, and capacitance per unit area, $C_{a}\left[\mathrm{~F} / \mathrm{cm}^{2}\right]$. Equation (5) [17] shows the final result:

$$
V_{P}=\frac{I_{0} R_{S}}{2 \pi} e^{j \omega t} K_{0}\left((1+j) \frac{r}{\delta}\right), \quad \delta=\sqrt{\frac{2}{\omega R_{S} C_{a}}}
$$

where $K_{0}(z)$ is the modified Bessel function of second kind and argument $z$ [18], and the characteristic length $\delta$ is identified as the diffusion length of the signal in the plane. Expression (5) gives the potential at $P$ as a time harmonic function whose attenuation with distance depends on the fraction $r / \delta$. This equation can be simplified in the following two limiting cases.

The first is when $r / \delta \rightarrow 0$, that is, we consider only distances $r$ from the origin much smaller than the diffusion length $\delta$. In this case, the following asymptotic expression for $K_{0}(z)$ holds [18]:

$$
K_{0}\left((1+j) \frac{r}{\delta}\right) \rightarrow \ln \frac{\delta \sqrt{2}}{r}-j \frac{\pi}{4}
$$

The second limiting case is the opposite. If $r / \delta \rightarrow \infty$, that is, the distance from the origin is large compared with $\delta$, or, for a given distance, the signal frequency is very high, then the following asymptotic expression holds [18]:

$$
K_{0}\left((1+j) \frac{r}{\delta}\right) \rightarrow \sqrt{\frac{\pi}{4}} \frac{\delta}{r} e^{-\frac{r}{\delta}} e^{-j\left(\frac{r}{\delta}+\frac{\pi}{4}\right)}
$$

Both cases are significant for the purpose of this paper. For instance, if the plane dimensions are much smaller than the diffusion length, the static analysis of Section 2 holds. A steady-state sinusoidal signal injected at a point reaches a receiver placed at any other point in the finite plane substantially with the same amplitude as when it is transmitted. On the contrary, if the dimensions of the finite plane are large compared with $\delta$, the transmitted signal damps exponentially with distance and is received only at a small neighborhood of the transmitter. Remember that $\delta$ depends on the frequency. Thus, given the plane dimensions, this parameter determines the maximum frequency of the signal that can be transmitted through the whole plane with low attenuation.

\section{B. Solution for a rectangle}

From expression (5) and by using the method of images it is possible to obtain a general expression for the potential measured at any point in a finite plane of dimensions $L \times H$. This potential can be calculated by adding the contribution of the currents at the image points of an infinite set of adjacent planes, all of dimensions $L \times H$.

According to (5), it is clear that the potential at $P$ is the sum of the current contribution and of all its images, as expression (8) shows:

$$
V_{P}=\frac{I_{0} R_{S}}{2 \pi} e^{j \omega t} \sum_{n, m=-\infty}^{+\infty} K_{0}\left(\frac{r_{n m}}{\delta^{\prime}}\right), \quad \delta^{\prime}=\frac{\delta}{1+j}
$$

where $r_{n m}$ is the distance between $P$ and image $n m$.

The previous analysis solves the problem for the case of a transmitter connected at one point in a plane with one or two conductive surfaces. The analysis is now extended to the case of a transmitter connected at two points in a plane with only one conductive surface (Figure 1).

We start with expression (8), which shows the potential at a point $P$ in a finite plane caused by a current source $I_{0}$ injected at a given point, for example T1. By superposition, we obtain the potential at $P$ when there is a current sink of value $-I_{0}$ at another point, for example T2. Using the same technique, we obtain the potential at a point $N$ produced by the same current source and sink. The points of application of the current source/sink are the locations of the transmitter dipole contacts, T1 and T2, and points $P$ and $N$ are the locations of the receiver dipole contacts, R1 and R2. Following this process, for the voltage at the receiver dipole $V_{R}=V_{P}-V_{N}=V_{R 1}-V_{R 2}$ we have

$$
V_{R}=\frac{I_{0} R_{S}}{2 \pi} e^{j \omega t}\left\{\sum_{n, m=-\infty}^{+\infty}\left[\left(K_{0}\left(\frac{r_{n m, p p}}{\delta}\right)+K_{0}\left(\frac{r_{n m, n n}}{\delta}\right)\right)-\left(K_{0}\left(\frac{r_{n m, n p}}{\delta}\right)+K_{0}\left(\frac{r_{n m, p p}}{\delta}\right)\right)\right]\right\}
$$

where $r_{n m, i j}$ denotes the distance between the point $i$ of the image current $\mathrm{nm}$ and the contact $j$ of the receiver dipole, 
being $i \in\{p \equiv \mathrm{T} 1, n \equiv \mathrm{T} 2\}$ and $j \in\{p \equiv \mathrm{R} 1, n \equiv \mathrm{R} 2\}$.

\section{EXPERIMENTAL RESULTS}

This Section presents several experiments with planes of different sizes and electrical parameters to check the theory in Sections II and III. Table I summarizes the dimensions and parameters of three of these planes.

TABLE I.

\begin{tabular}{|l|l|l|l|l|l|l|}
\hline $\begin{array}{l}\text { Plane } \\
\#\end{array}$ & $\begin{array}{l}\boldsymbol{L} \\
{[\mathbf{c m}]}\end{array}$ & $\begin{array}{l}\boldsymbol{W} \\
{[\mathbf{c m}]}\end{array}$ & $\begin{array}{l}\boldsymbol{R}_{\boldsymbol{s}} \\
{[\boldsymbol{\Omega} / \square]}\end{array}$ & $\begin{array}{l}\boldsymbol{C}_{\boldsymbol{a}} \\
{\left[\mathbf{F} / \mathbf{c m}^{2}\right]}\end{array}$ & $\begin{array}{l}\# \text { of } \\
\text { cond. } \\
\text { surf. }\end{array}$ & Case \\
\hline 1 & 140 & 73.6 & 1462 & $163 \times 10^{-15}$ & 2 & Dynamic \\
\hline 2 & 73.6 & 54.4 & 1462 & $14 \times 10^{-15}$ & 1 & $\begin{array}{l}\text { Static/Dyna } \\
\text { mic }\end{array}$ \\
\hline 3 & 210 & 122 & 528 & $9.3 \times 10^{-15}$ & 1 & $\begin{array}{l}\text { Static/Dyna } \\
\text { mic }\end{array}$ \\
\hline
\end{tabular}

\section{A. Results for plane \#1}

This plane consists of a pair of sheets of conductive paper adhered to both sides of a $0.5 \mathrm{~cm}$ thick, $140 \mathrm{~cm}$ long, $73.6 \mathrm{~cm}$ wide foam plate. That is, this plane has two conductive surfaces. A pair of contacts (one at each side) in the center of each sheet connects the transmitter. The measurement points (receiver contacts) are at fifteen places distributed in the following way: seven points separated by $10 \mathrm{~cm}$ and placed between the center and the short side of the rectangle (horizontal points); five contacts separated by $10 \mathrm{~cm}$ and placed in diagonal (diagonal points); and three contacts separated by $10 \mathrm{~cm}$ and placed between the center and the long side of the rectangle (vertical points). All contacts are connected to both sides of the plane by circular copper discs with a radius of $1.3 \mathrm{~cm}$.

An Agilent 33120A Function Generator provides the transmitter contacts with a sinusoidal voltage with a constant amplitude of $1 \mathrm{Vpp}$ at the following twelve frequencies: $1 \mathrm{KHz}$, $10 \mathrm{KHz}, 20 \mathrm{KHz}, 50 \mathrm{KHz}, 100 \mathrm{KHz}, 200 \mathrm{KHz}, 500 \mathrm{KHz}, 1 \mathrm{MHz}$, $2 \mathrm{MHz}, 5 \mathrm{MHz}, 10 \mathrm{MHz}$ and $15 \mathrm{MHz}$. Two differential probes Tektronix P6247 connected to a TEKTRONIX TDS7000 Digitizing Oscilloscope measure both the signal amplitude at the centered contacts and the amplitude at the other receiver contacts.

The solid lines in Figure 3 represent the amplitudes at the horizontal points calculated with expression (8), and the circles represent the measured amplitudes. The upper and decay lines correspond to the lower and higher frequencies, respectively. Results for the diagonal and vertical points are similar. As can be seen, the agreement is very good for the lower and higher frequencies and less good but still remarkable for the $50 \mathrm{KHz}, 100 \mathrm{KHz}$ and $200 \mathrm{KHz}$ frequencies.

It is interesting to observe how the voltage at the transmitter contacts (circles in the top left corner of Figure 3, is not constant but depends on the frequency. This phenomenon is explained by considering the two conductive surfaces as the plates of a capacitor. The signal from the Function Generator is applied to a capacitive load, so its internal output impedance
$(50 \Omega)$ and the capacitor form a frequency-dependent voltage divider that attenuates the effective signal amplitude at the transmitter contacts.

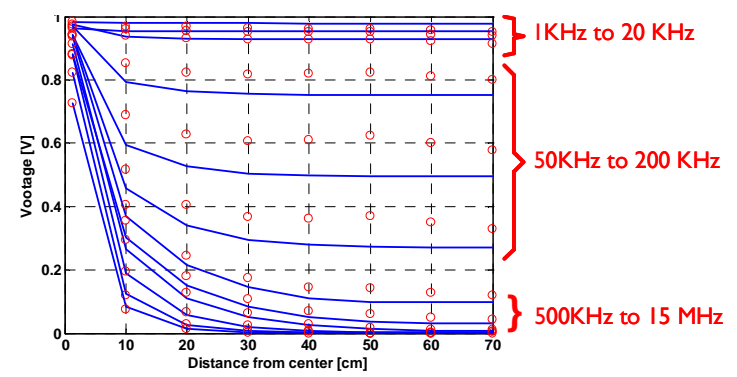

Fig. 3. Voltage amplitudes at the seven horizontal points of plane \#1. Solid lines: calculated voltage at each point, circles: measured.

Also note the correctness of the analysis in the previous section. At high frequency (small diffusion length $\delta$ ) the signal is strongly attenuated with distance whereas at low frequency (large diffusion length) the attenuation is small and the signal received at any place has practically the same amplitude as the transmitted signal.

\section{B. Results for plane \#2}

This plane consists of a $73.6 \mathrm{~cm}$ long, $54.4 \mathrm{~cm}$ wide single sheet of conductive paper lying on top of a wooden platform. The contacts of the transmitter dipole are copper discs with a radius of $1.3 \mathrm{~cm}$ placed near the corners of one of the short sides of the plane and separated by $40.8 \mathrm{~cm}$. The contacts of the receiver dipole are made up of a pair of copper discs with a radius of $0.3 \mathrm{~cm}$ separated by $1.8 \mathrm{~cm}$. The signal received by the dipole is measured at 49 places equally distributed over the plane.

For the static measurements, a constant voltage source is connected to the transmitter dipole contacts and a voltmeter measures the voltage at the receiver dipole. For the dynamic measurements, as in plane \#1, the sinusoidal input signal is generated by the Function Generator with a constant amplitude of $1.8 \mathrm{Vpp}$ at the following frequencies: $1 \mathrm{KHz}, 10 \mathrm{KHz}, 20$ $\mathrm{KHz}, 50 \mathrm{KHz}, 100 \mathrm{KHz}, 200 \mathrm{KHz}, 500 \mathrm{KHz}, 1 \mathrm{MHz}, 2 \mathrm{MHz}$, $5 \mathrm{MHz}, 10 \mathrm{MHz}$ and $15 \mathrm{MHz}$. The differential probes simultaneously measure the voltage amplitude at the transmitter dipole and at the receiver dipole.

\section{1) Static measurements}

In the static measurements, the transmitter dipole is connected to a constant voltage source of $10 \mathrm{~V}$ injecting a constant current of $3.03 \mathrm{~mA}$. A voltmeter measures the potential difference between the pair of contacts of the receiver dipole. Two series of measurements reveal the influence of the orientation of the axis of the receiver dipole with respect to the axis of the transmitter dipole. The first series shows the voltage at the receiver dipole when it is oriented in the same direction as the transmitter dipole, that is, they are parallel. The second series shows the voltage at the receiver dipole when it is perpendicular to the transmitter 
dipole. We call the first orientation parallel and the second perpendicular. Figures 4 and 5 summarize the results of both series.

Figure 4 is a logarithmic plot of the voltage at the receiver dipole for the $7 \times 7=49$ points on the surface for parallel orientation. In this case, the voltage is always positive. The filled surface is the value calculated from formula (4) and the dots represent the measured values. Notice that the agreement is very good.

Figure 5 provides the same results for perpendicular orientation. Now the voltage is positive or negative depending on the measurement point. The filled surface shows the calculated values and the dots the measured ones. Again, the agreement is excellent. This illustrates the importance of the relative orientation of transmitter and receiver dipoles. In these Figures the $\mathrm{X}$ and $\mathrm{Y}$ axis are the indices of the $7 \mathrm{x} 7$ places of the measuring points.

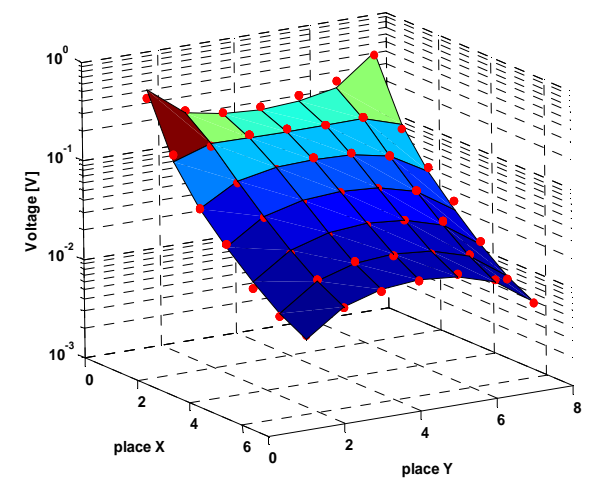

Fig. 4. Voltage at the receiver for static measurements on plane \#2. Parallel orientation. Filled surface: calculated from (4), dots: measured.

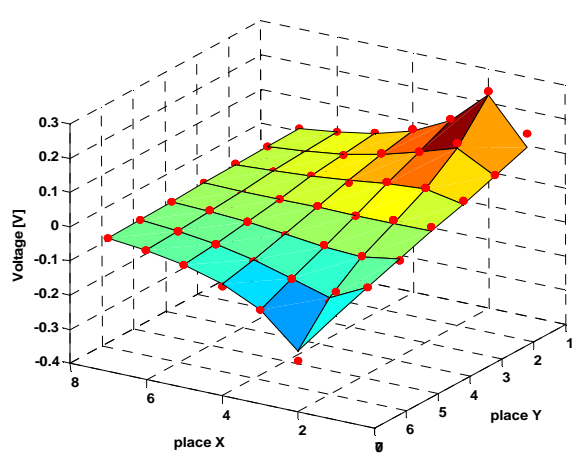

Fig. 5. Voltage at the receiver for static measurements on plane \#2.

Perpendicular orientation. Filled surface: calculated from (4), dots: measured.

\section{2) Dynamic measurements}

In the dynamic measurements, the Function Generator is connected at the same place as the voltage source in the static measurements. The amplitude of the generated voltage is $1.8 \mathrm{Vpp}$. A differential probe connected to the oscilloscope measures the signal amplitude at the receiver dipole. Both dipoles have parallel orientation.

Figure 6 compares measured and calculated values at each of the 49 measurement points at $5 \mathrm{MHz}$. The squares and the circles represent the values calculated by expression (9) and the measured values, respectively. The response at other frequencies is similar to that shown in this Figure. Again, $\mathrm{X}$ and $\mathrm{Y}$-axis are the indices of the $7 \times 7$ measuring points.

As can be seen, the agreement is very good for most points. The differences between calculations and measurements can be explained by the non-uniformities of the electrical parameters and unavoidable uncertainties in the measurement of low-level signals. The relatively large deviation at the farthest points observed in Figure 6 is due to the small amplitude of the receiver signal (less than $1 \mathrm{mV}$ ), which is lower than the electrical noise captured by the oscilloscope, whose averaging and synchronizing capabilities were stretched to the limit.

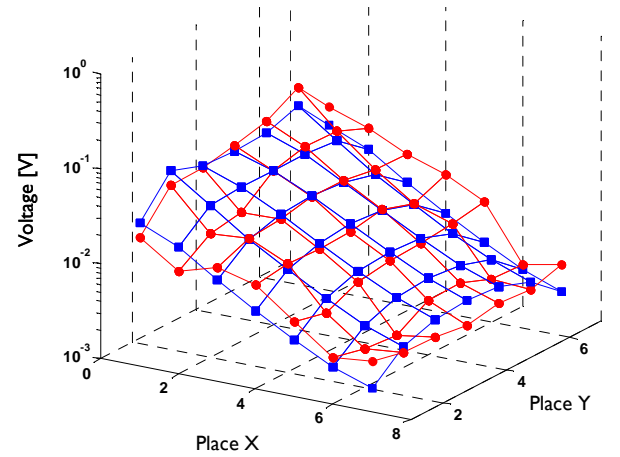

Fig. 6. Voltage amplitudes calculated (squares) and measured (circles) on plane $\# 2$ at $5 \mathrm{MHz}$.

\section{Results for plane \#3}

Plane \#3 consists of a $2 \mathrm{~cm}$ thick, $210 \mathrm{~cm}$ long, $122 \mathrm{~cm}$ wide wooden platform covered with a layer of conductive paint. The transmitter dipole is close to the two corners of one of the short sides of the plane. As in planes \#1 and \#2, the transmitter contacts are circular copper discs with a radius of $1.3 \mathrm{~cm}$. For plane \#3 only dynamic measurements are shown.

In this case, the waveform supplied by the Function Generator is a rectangular signal of frequency $100 \mathrm{KHz}$ and $5 \mathrm{Vpp}$ amplitude. A differential probe connected to the oscilloscope measures the signal amplitude at the receiver dipole.

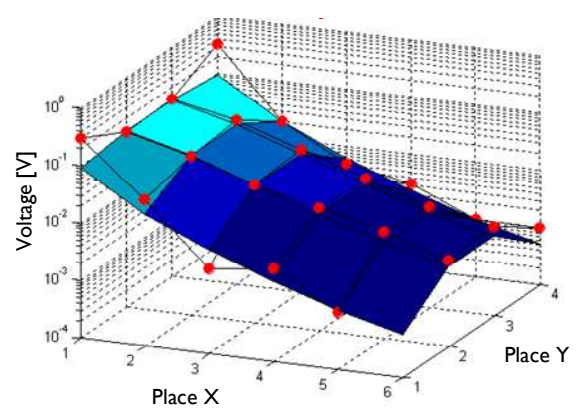

Fig. 7. Voltages measured and calculated on plane \#3 with parallel orientation of dipoles.

Figures 7 and 8 compare values measured and calculated at each of the $6 \times 4=24$ measurement points for parallel and perpendicular orientation of dipoles, respectively. As far as perpendicular orientation is concerned, Figure 8 shows the 
absolute value of the voltage at the receiver dipole. The circles and the filled surfaces are measured values (in V) and values calculated by expression (9), respectively.

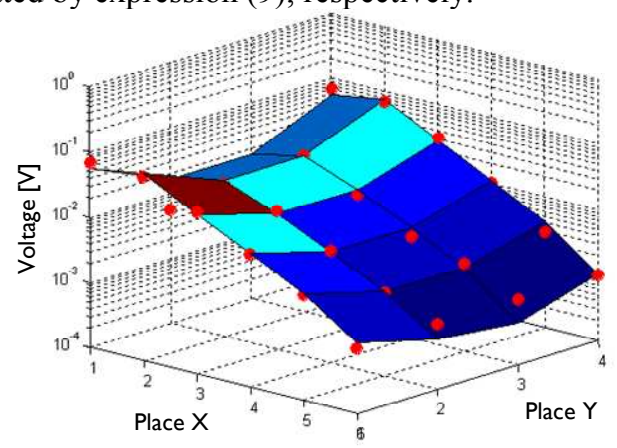

Fig. 8. Absolute value of voltage measured and calculated on plane \#3 with perpendicular orientation of dipoles.

The agreement between measured and calculated values is very good, thus supporting the correctness of the analyses in Sections 2 and 3.

\section{Prototype of communication network using only one} conductive surface

We built a prototype of a communication system as an experimental platform to check the validity of our proposal. It is currently composed of transceivers connected to a PC and communicated through the conductive surface of plane \#3 at $100 \mathrm{Kbit} / \mathrm{s}$. Standard protocols are used in a half duplex bidirectional communication. Figure 9 shows a photograph of the test bench.

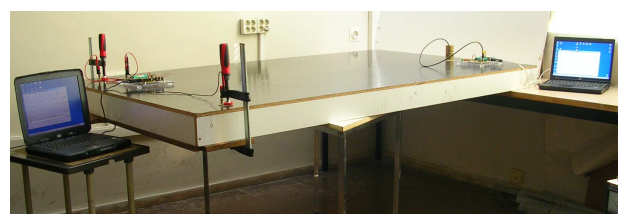

Fig. 9. Test bench of a prototype of a communication system using a single conductive surface. Left: transmitter, right: receiver.

\section{MAXIMUM FREQUENCY, DISTANCE AND POWER CONSUMPTION}

This Section analyzes the relationships between the size and material properties of planes with only one conductive surface, and important characteristics of any transmission system, such as maximum speed available, maximum distance between transmitters and receivers and power/energy consumption of devices. First, we examine the relationships between the current injected into the surface by the transmitter and its sheet resistance. Both magnitudes are related to power consumption. Next, we explore the relationships between the transmitter and the receiver dipole dimensions, distance between them and maximum speed available.

\section{A. Current, sheet resistance and power consumption}

It is clear that the resistivity of materials used to cover the transmission surface strongly influences the features of the transmission system. On the one hand, if the resistivity is very high (surfaces with very high sheet resistance), nothing can be transmitted because the current injected by the transmitter becomes zero. On the other hand, materials like metals with very low resistivity (surfaces with very low sheet resistance) are not particularly useful for transmission either, because they behave as short-circuits, thus overloading the transmitter circuits. However, using the proposed approach a range of values for surface sheet resistance becomes suitable for communication. This range is not analyzed in this paper. Instead, this sub-section presents some measured results obtained from surfaces with different sheet resistances.

Equations (4) and (9) show that the voltage at the receiver dipole is proportional to the product $I R_{S}$, where $I$ is the current injected by the transmitter and $R_{S}$ is the sheet resistance. In fact, the transmitter signal is usually injected into the conductive surface as a voltage $V_{0}$ applied to the transmitter connections. Thus, by Ohm's law current $I$ is $I=V_{0} / R_{T}$, where $R_{T}$ is the load resistance as seen from the transmitter contacts which in turn is proportional to $R_{S}$. Thus, if we measure $R_{T}$, expressions (4) and (9) can be written as a function of $V_{0}$ instead of $I$. Let us now look at some experimental relations between $R_{S}, V_{0}$ and power consumption, and at an estimation of the total energy consumed in the transmission.

Tables II and III summarize these results. The first and second columns identify the measured plane and its size. Planes \#2 and \#3 are the same as in Section IV. Plane \#4 is of a similar size to that of plane \#3, but the former is painted with a more conductive paint. The third column shows the sheet resistance of the conductive surface. The fourth column is the measured resistance between the transmitter contacts, $R_{T}$. The fifth column shows the voltage $V_{0}$ applied to the transmitter contacts in the static/dynamic measurements. The sixth column is the resulting current injected by the transmitter $I_{T}$, and the seventh contains the power supplied by the power supply, $P_{T}=V_{0} \times I_{T}$. Tables II and III show the above data for the static and dynamic experiments respectively.

TABLE II.

\begin{tabular}{|l|l|l|l|l|l|l|}
\hline $\begin{array}{l}\text { Plane } \\
\#\end{array}$ & $\begin{array}{l}\boldsymbol{L} \times \boldsymbol{W} \\
{[\mathbf{c m}]}\end{array}$ & $\begin{array}{l}\boldsymbol{R}_{\boldsymbol{S}} \\
{[\boldsymbol{\Omega} / \square]}\end{array}$ & $\begin{array}{l}\boldsymbol{R}_{\boldsymbol{T}} \\
{[\boldsymbol{\Omega}]}\end{array}$ & $\begin{array}{l}\boldsymbol{V}_{\mathbf{0}} \\
{[\mathbf{V}]}\end{array}$ & $\begin{array}{l}\boldsymbol{I}_{\boldsymbol{T}} \\
{[\mathbf{m A}]}\end{array}$ & $\begin{array}{l}\boldsymbol{P}_{\boldsymbol{T}} \\
{[\mathbf{m W}]}\end{array}$ \\
\hline 2 & $73.6 \times 54.4$ & 1462 & 3300 & 10 & 3.03 & 30.3 \\
\hline 3 & $210 \times 122$ & 528.7 & 255 & 5 & 19.6 & 98 \\
\hline 4 & $244 \times 122$ & 77 & 43 & 2.2 & 50.4 & 109 \\
\hline
\end{tabular}

TABLE III.

\begin{tabular}{|l|l|l|l|l|l|l|}
\hline $\begin{array}{l}\text { Plane } \\
\#\end{array}$ & $\begin{array}{l}\boldsymbol{L} \times \boldsymbol{W} \\
{[\mathbf{c m}]}\end{array}$ & $\begin{array}{l}\boldsymbol{R}_{\boldsymbol{S}} \\
{[\boldsymbol{\Omega} / \square]}\end{array}$ & $\begin{array}{l}\boldsymbol{R}_{\boldsymbol{T}} \\
{[\boldsymbol{\Omega}]}\end{array}$ & $\begin{array}{l}\boldsymbol{V}_{\mathbf{0}} \\
{[\mathbf{V p p}]}\end{array}$ & $\begin{array}{l}\boldsymbol{I}_{\boldsymbol{T}} \\
{[\mathbf{m A} \mathbf{A}]}\end{array}$ & $\begin{array}{l}\boldsymbol{P}_{\boldsymbol{T}} \\
{[\mathbf{m W}]}\end{array}$ \\
\hline 2 & $\begin{array}{l}73.6 \times 54 . \\
4\end{array}$ & 1462 & 3300 & $1.8^{*}$ & 0.55 & $0.98^{* *}$ \\
\hline 3 & $210 \times 122$ & 528.7 & 255 & $5^{*}$ & 19.6 & 98 \\
\hline 4 & $244 \times 122$ & 77 & 43 & $1.6^{*}$ & 37.2 & $59.5^{* *}$ \\
\hline
\end{tabular}

* in planes \#2 and \#4 the applied signal is sinusoidal, so we take the amplitude. In plane \#3 the signal is rectangular.

** this number is the maximum instantaneous power; the average power value is lower.

\section{B. Time and energy estimation}

From the data of Table III and by assuming a given transmission speed, we can estimate the energy consumed per 
transmitted bit as ET $=\mathrm{PT} \times \mathrm{T}$, where $\mathrm{ET}$ is the energy in $\mathrm{J}$ and $\mathrm{T}$ is the time of one bit in seconds. Therefore, assuming, for instance, that a digital signal of $5 \mathrm{~V}$ amplitude is transmitted at a speed of $100 \mathrm{Kbit} / \mathrm{s}$, we have the energy per bit, as shown in Table IV for the tested planes.

TABLE IV.

\begin{tabular}{|l|l|l|}
\hline Plane \# & $\boldsymbol{P}_{\boldsymbol{T}}[\mathbf{m W}]$ & Energy per bit $[\boldsymbol{\mu J}]$ \\
\hline 2 & 7.6 & 0.076 \\
\hline 3 & 98 & 0.98 \\
\hline 4 & 581 & 5.81 \\
\hline
\end{tabular}

The total energy spent in sending a message depends on the number of bits of the message. If the message length is, say, 100 bits, the energy consumption per message is between 7.6 and $581 \mu \mathrm{J}$. In the worst case, by taking $1 \mathrm{~mJ}$ as the limit of the energy spent per message by a transmitter connected to plane $\# 4$, about $10^{6}$ messages can be sent before fully discharging an off-the-shelf AA battery for portable devices. This number may be acceptable for many applications. Of course, if planes \#2 or \#3 are used, the number of messages increases by orders of magnitude.

The transceiver has other sources of power consumption when it is operating or in standby, implying that low power digital design techniques must be used to reduce these contributions to a minimum. In addition, when no data are transmitted, specific circuits connected to the plane should restore the energy lost by the transmitters. Nevertheless, the purpose of this sub-section is only to show the feasibility of transmitting data with an acceptable low power consumption using a single conductive surface. For this reason, techniques for low power circuit design or for remote power transmission are not analyzed here.

\section{Size, distance and orientation of transmitter/receiver}

Equations (4) and (9) determine the relationships between the distance and size of the transmitter/receiver dipoles and the amplitude of the received signal. These relationships are contained in the arguments of the theta functions in (4) and the Bessel functions in (9), but these expressions are too complex to allow direct conclusions to be drawn. Instead, an approximate interpretation obtained from these equations and confirmed by the experiments on the tested planes is given next.

If the signal frequency is sufficiently low (which means $r<<\delta$, see Section III), the amplitude of the received signal $V_{R}$ can be calculated from (4). The following rules hold for the amplitude of the received signal in these conditions:

(i) If the distance between the transmitter contacts $D_{T}$ or the receiver contacts $D_{R}$ is of the order of magnitude of the plane size, then $V_{R}$ approximately decreases exponentially with the distance between the centers of the transmitter and receiver dipoles, $d_{T R}$. However, if the effect of the boundaries is neglected (the plane can be considered as infinite) and $D_{T}+D_{R}$ $<<d_{T R}$, then the decrease is only approximately quadratic with $d_{T R}$. (ii) $V_{R}$ is approximately proportional to the product $D_{T}$ $\times D_{R}$. That is, doubling the distance between the transmitter or receiver contacts, the amplitude of the received signal is roughly doubled. Consequently, in the tested planes the contacts of the transmitters are well separated and placed close to the corners of one side of the planes. (iii) $V_{R}$ reaches its maximum value at a given point on the surface when the receiver dipole is oriented in the same direction as the maximum gradient of the potential field created by the transmitter at that point. (iv) The reciprocity theorem holds, and therefore it is possible a bidirectional communication between the networked devices.

Let us examine the results obtained from measurements on the tested planes. Table $\mathrm{V}$ identifies the planes and summarizes their electrical parameters in columns 1-3. Column 4 lists the distance between the transmitter contacts $D_{T}$ and their radius $r_{T}$. Column 5 gives the above parameters for the receiver dipole, $D_{R}$ and $r_{R}$. Column 6 contains the distance between the centers of the transmitter and receiver dipoles $d_{T R}$, and column 7 shows the amplitude of the voltage measured at the receiver dipole $V_{R}$. The dipole axes are oriented in parallel and the transmitter is connected at the same points as in the experiments of Section 4. The frequency is $100 \mathrm{KHz}$, which guarantees that all distances are well below $\delta$. The amplitude of the transmitted signal to each plane, $V_{0}$, is the same as in Table III.

TABLE V

\begin{tabular}{|l|l|l|l|l|l|l|}
\hline $\begin{array}{l}\text { Plane } \\
\#\end{array}$ & $\begin{array}{l}\boldsymbol{R}_{S} \\
{[\boldsymbol{\Omega} / \square]}\end{array}$ & $\begin{array}{l}\boldsymbol{C}_{\boldsymbol{a}} \\
{\left[\mathbf{f F} / \mathbf{c m}^{2}\right]}\end{array}$ & $\begin{array}{l}\boldsymbol{D}_{T}, \boldsymbol{r}_{T} \\
{[\mathbf{c m}]}\end{array}$ & $\begin{array}{l}\boldsymbol{D}_{R}, \boldsymbol{r}_{R} \\
{[\mathbf{c m}]}\end{array}$ & $\begin{array}{l}\boldsymbol{d}_{T R} \\
{[\mathbf{c m}} \\
]\end{array}$ & $\begin{array}{l}\boldsymbol{V}_{R} \\
{[\mathbf{m V}]}\end{array}$ \\
\hline 2 & 1462 & 14 & $40.8,1.3$ & $1.8,0.3$ & 54 & 1.4 \\
\hline 3 & 528.7 & 9.3 & $105,1.3$ & $3.6,0.3$ & 198 & 3.65 \\
\hline 4 & 77 & 10.1 & $108,1.3$ & $1.8,0.3$ & 228 & 0.71 \\
\hline
\end{tabular}

It can be observed that, for similar $d_{T R}$ and $D_{T}$, the amount of received signal $V_{R}$ is about five times larger in plane \#3 than in plane \#4. This is mainly due to the combination of greater $V_{0}$ and $D_{R}$ in plane \#3. Also, note that planes \#2 and \#4 have similar $V_{0}$ and the same $D_{R}$. They have different $D_{T}$ and $d_{T R}$ but, comparatively, the transmitter and the receiver are closer in plane \#2 than in plane \#4, so $V_{R}$ is greater in plane \#2.

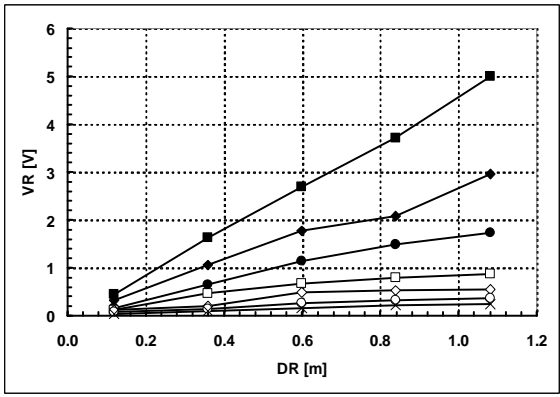

Fig. 10. $V_{R}$ as a function of $D_{R}$ taking $d_{T R}$ as a parameter. Plane \#3. Upper line: $d_{T R}=0 \mathrm{~m}$, lower line: $d_{T R}=1.44 \mathrm{~m}$.

Figures 10 and 11 show how received voltage obeys rules (i) and (ii). The $V_{R}$ measured in plane \#3 is shown as a function of $D_{R}$ and $d_{T R}$. The transmitter is connected near the 
corners of one short side of plane \#4 and $D_{T}=105 \mathrm{~cm}$. The frequency is $100 \mathrm{KHz}$ and $V_{0}=5 \mathrm{Vpp}$. The dipole axes are oriented in parallel.

Figure 10 plots $V_{R}$ as a function of $D_{R}$ for seven equally spaced values of $d_{T R}$ from 0 to $1.44 \mathrm{~m}$. As can be seen, there is a practically linear relation between $V_{R}$ and $D_{R}$. Figure 11 illustrates the dependency of $V_{R}$ on $d_{T R}$ for five equally spaced distances $D_{R}$ from $0.12 \mathrm{~m}$ to $1.08 \mathrm{~m}$. Here, the exponential decrease of $V_{R}$ is clearly observed.

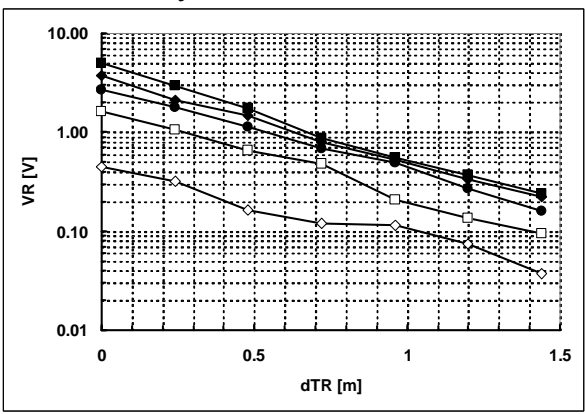

Fig. 11. $V_{R}$ as a function of $d_{T R}$ taking $D_{R}$ as a parameter. Plane \#3. Upper line: $D_{R}=1.08 \mathrm{~m}$, lower line: $D_{R}=0.12 \mathrm{~m}$.

\section{CONCLUSIONS}

The purpose of this paper is to show how data transmission between networked devices is possible by using only a single conductive surface. The amplitude of the received signal and the transmission speed in rectangular planes with one or two conductive surfaces are analyzed as a function of electrical parameters and dimensions. Measurements on planes with a size between $73.6 \times 54.4 \mathrm{~cm}$ and $244 \times 122 \mathrm{~cm}$ and with sheet resistances from 77 to $1462 \Omega$ /square corroborate the validity of the analysis. Practical rules to locate and orient the transceivers are given. Successful experiments demonstrate the feasibility of communications between networked devices using a single conductive surface with prototype transceivers, which can communicate at $100 \mathrm{Kbit} / \mathrm{s}$ at a distance of $2 \mathrm{~m}$ in a plane of $2.56 \mathrm{~m}^{2}$. Issues as remote power transmission or contact less coupling of transceivers to the conductive surface are on going work not covered in this paper. Potential applications of this approach are in the fields of surface testing, home automation and wearable computing.

\section{ACKNOWLEDGMENT}

The author would like to thank students Vicenç Casadevall and Francesc Bonjoch for their help in making measurements on planes \#2, \#3 and \#4.

\section{REFERENCES}

[1] F. Scott, F. Hoffmann, M. Adledsee, G. Mapp, A. Hopper, "Networked Surfaces: a New Concept in Mobile Networking, Proceedings of Third IEEE Workshop on Mobile Computing Systems and Applications, IEEE, December 2000.

[2] K. Van Laerhoven, N. Villar, A. Schmidt and H.-W. Gellersen, "Pin@Play: The Surface as Network Medium", IEEE Communications Magazine, April 2003, pp. 90-95.
[3] K.F. Laerhoven, "The Pervasive Sensor", Proceedings of Second International Conference on Ubiquitous Computing Systems, UCS2004, November 2004, pp. 1-5.

[4] J. Lifton, J.A. Paradiso, "Pushpin Computing System Overview: A Plattform for Distributed, Embedded, Ubiquitous Sensor networks", Proceedings of Pervasive Computing, LNCS 2414, Springer Verlag, 2002, pp139-151.

[5] J. Lifton, M. Broxton, J.A. Paradiso, "Experiences and Directions in Pushpin Computing", Proceedings of $4^{\text {th }}$ International Conference on Information Processing in Sensor Networks, IPSN2005, April 2005, paper 57.

[6] H. Shinoda, N. Asamura, M. Hakozaki, X. Wang, "Two-Dimensional Signal Transmission Technology for Robotics", Proceedings of the 2003 International Conference on Robotics and Automation, pp. 32073212 , September 2003

[7] Y. Makino, K. Minamizawa, H. Shinoda, "Two Dimensional Communication Technology for Networked Sensing System", Proceedings of International Workshop on Networked Sensing Systems 2005 , pp. 168-173, June 2005.

[8] H. Shinoda, Y. Makino, N. Yamahira, H. Itai, "Surface Sensor Network Using Inductive Signal Transmission Layer", Proceedings of International Conference on Networked Sensing Systems 2007, pp. 201206, June 2007.

[9] Y. Makino, S. Ogawa, H. Shinoda, "EMG Sensor Integration Based on Two-Dimensional Communication", Proceedings of International Conference on Networked Sensing Systems 2008, pp. 168-173, June 2008.

[10] Van Laerhoven, et. al., "A Layered Approach to Wearable Textile Networks", Proceedings of IEEE Eurowearable Workshop, 2003.

[11] J. Akita, T. Shinmura, M. Toda, "Flexible Network System for Wearable Computing Using Conductive Fabric", Proceedings of the 7th International Conference on Mobile Data Management, MDM2006, pp. 101-104.

[12] E. Wade, H. Asada, "Conductive-Fabric Garment for a Cable-Free Body Area network", IEEE Pervasive Computing Magazine, January-March 2007, pp. 52-58.

[13] J. Akita, T. Shinmura, S. Sakurazawa, K. Yanagihara, M. Kunita, M. Toda, K. Iwata, "Wearable electromyography measurement system using cable-free network system on conductive fabric", Artificial Intelligence in Medicine, (2008) Vol. 42, pp. 99-108

[14] E.R. Wade, H. Harry Asada, "DC Powerline Communication Network for a Wearable Health Monitoring System", Proceeedings of ISPLC 2005 , pp. $172-175$.

[15] J.W. Ward, R.V. Churchill, Complex variables and applications, 7th edition, McGraw Hill, New York, 2004

[16] E.T. Whittaker and G.N. Watson, A course of modern analysis, 4th edition, Cambridge University Press, 1927.

[17] H.S. Carslaw and J.C. Jaeger, Conduction of heat in solids, second edition, Oxford Unversity Press, Osford, 1959.

[18] G.N. Watson, A treatise on the theory of Bessel functions, second edition, page 182, Cambridge University Press, 1966. 